\title{
V.11 Compatibility of Antiwear Additives with Non-Ferrous Engine Bearing Alloys
}

\section{Overall Objectives}

- Investigate the compatibility of engine lubricant antiwear (AW) additives, specifically conventional zinc dialkyldithiophosphate (ZDDP) and newly developed ionic liquids (ILs), with selected non-ferrous engine bearing alloys, specifically aluminum and bronze alloys that are commonly used in connecting rod end journal bearings and bushings, to gain fundamental understanding to guide future development of engine lubricants

\section{Fiscal Year (FY) 2016 Objectives}

- Investigate the boundary friction and wear behavior of selected Al-Si and bronze alloys in ZDDP- and ionicliquid-additized lubricants

- Gain initial understanding of the interactions between the $\mathrm{AW}$ additives and non-ferrous alloys via tribofilm characterization

\section{FY 2016 Accomplishments}

- Conducted a series of boundary lubrication tribo-tests on Al-Si and bronze alloys against steel using ZDDPand ionic-liquid-additized lubricants

- Friction and wear behavior largely depends on the AWmaterial combination; Al 380 alloy, ZDDP, phosphate IL, carboxylate IL, and mixture of ZDDP and phosphate or sulfonate IL showed effective wear protection; in contrast, sulfonate IL and mixture of ZDDP and carboxylate IL had a significant detrimental impact with the wear rate increased by two orders of magnitude

- Performed tribofilm morphology examination and composition analysis to seek fundamental understanding of the lubricant-material compatibility

\section{Future Directions}

- Continue to explore the effectiveness of AW additives on bronze in boundary lubrication

- Understand the mechanisms behind the detrimental impact observed on some AW additives when lubricating $\mathrm{Al}-\mathrm{Si}$ and bronze alloys

- Expand the compatibility study to friction modifiers interacting with $\mathrm{Al}-\mathrm{Si}$ and bronze alloys in mixed and moderate boundary lubrication regimes

\author{
Jun Qu (Primary Contact) and \\ Yan Zhou \\ Oak Ridge National Laboratory \\ Materials Science \& Technology Division \\ P.O. Box 2008 \\ Oak Ridge, TN 37831-6063 \\ Phone: (865) 576-9304 \\ Email: qujn@ornl.gov \\ DOE Technology Development Manager: \\ Michael Weismiller
}

\section{Introduction}

Decreasing engine oil viscosity inevitably reduces the thickness of oil films at the engine bearing interfaces, a wear challenge with more frequent surface asperity collisions. Bronze- and aluminum-based alloys are commonly used in connecting rod end journal bearings and bushings. However, current engine lubricants and their additive packages were designed for ferrous alloys and their compatibility with non-ferrous alloys is not well understood. For instance, literature results of using ZDDP-containing oils lubricating aluminum alloys varied significantly in different testing conditions. One study reported effective wear reduction of aluminum in highload rolling contact when 5\% ZDDP was introduced to the oil [1]. In contrast, another report showed that the addition of 5\% ZDDP increased the wear rate of an $\mathrm{Al}$ alloy sliding against steel [2]. This project investigates the compatibility of engine lubricant AW additives, specifically conventional ZDDP and newly developed ILs, with selected non-ferrous engine bearing alloys, specifically aluminum and bronze alloys. Fundamental understanding gained in this study will help guide future development of engine lubricants.

\section{Approach}

Work scope includes: (1) tribosystem analysis of the engine bearing interfaces of interest to design the tribological bench tests, (2) investigation of the boundary friction and wear behavior for selected bronze and $\mathrm{Al}-\mathrm{Si}$ alloys lubricated by ZDDP- and ionic-liquid-additized lubricants, and (3) understanding the tribochemical interactions between the non-ferrous alloys and AW additives via tribofilm characterization. 
As shown in Figure 1, four AW additives were used in our study: a secondary ZDDP, a phosphonium-phosphate IL ([P $\left.{ }_{8888}\right][\mathrm{DEHP}],>10 \%$ oil solubility), a phosphoniumcarboxylate IL $\left(\left[\mathrm{P}_{66614}\right]\left[\mathrm{C}_{17} \mathrm{H}_{35} \mathrm{COO}\right],>10 \%\right.$ oil solubility), and a phosphonium-sulfonate IL ( $\left[\mathrm{P}_{66614}\right]$ $\left[\mathrm{RSO}_{3}\right], 1-2 \%$ oil solubility). All three ILs had previously shown effective wear protection for ferrous alloys [3]. Each additive was added into a SAE 0W-30 base oil at a treat rate to get a phosphorus content of $800 \mathrm{wt}$ ppm. When two additives were used together, each contributed 400 ppm P. Aluminum alloy 380 and bronze alloy C932 were rubbed against American Iron and Steel Institute 52100 steel bearing balls of $10 \mathrm{~mm}$ using a Plint TE77 tribometer. The reciprocating sliding tests were carried out at ambient environment under a $20 \mathrm{~N}$ load and a $10 \mathrm{~Hz}$ oscillation with a $10-\mathrm{mm}$ stroke for $200 \mathrm{~m}$ to $10,000 \mathrm{~m}$ sliding. The friction coefficient was captured in situ and wear volume was quantified using white interferometry. The worn surface morphology and composition were characterized using scanning electron microscopy (SEM) and energy-dispersive X-ray spectroscopy (EDS).

\section{Results}

\section{Investigation of the Compatibility Between AW and Aluminum Alloy}

Figure 2a shows the friction behavior of three 1,000-m tests of the steel-aluminum contact in the base oil and the surface morphology of the steel balls that rubbed against Al 380 flats. The worn surfaces on the steel balls showed clear signs of material transfer from the Al 380 flats. As highlighted in the friction plots, each test started with a period of a higher level of friction $(\sim 0.08)$ and dropped to a lower level $(<0.05)$ with a quick transition. The wear volume of the Al flats in the three tests were $0.082 \mathrm{~mm}^{3}$, $0.12 \mathrm{~mm}^{3}$, and $0.036 \mathrm{~mm}^{3}$, respectively, which seemed proportional to the span of the high-friction stage. A 200-m test was then performed to intentionally stop in the high-friction stage, and its friction trace is shown in Figure 2b. The SEM imaging and EDS elemental mapping indicated an Al- and O-rich transfer layer on the steel counterface. An extended test of 10,000-m sliding was also run, and the friction curve is shown in Figure 2c. The wear volumes for the 200-m test and the 10,000-m test were $0.014 \mathrm{~mm}^{3}$ and $0.022 \mathrm{~mm}^{3}$, respectively, indicating that most material loss occurred in the highfriction stage.

The friction traces of three repeat tests for each oil containing a single AW additive, ZDDP or IL with 800 ppm P content, are shown in Figure 3a. The wear volume of the $\mathrm{Al} 380$ flat in each individual test is marked in the legend. Average wear volumes are compared in Figure 3b. The addition of the ZDDP, phosphate IL or carboxylate IL either eliminated or reduced the length of the high-friction stage and the wear volume.

ZDDP
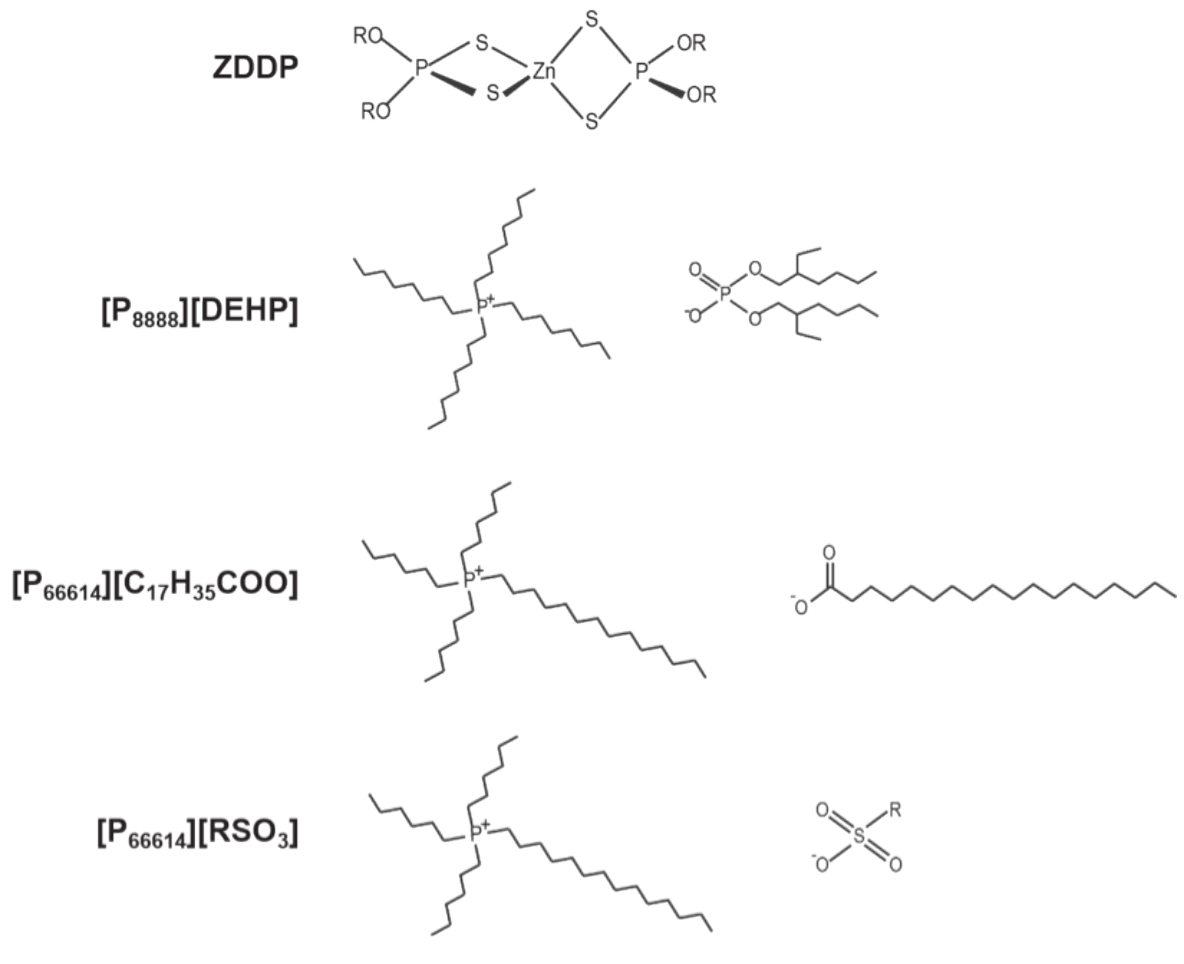

Figure 1. Molecular structures of ZDDP and three ILs used as AW additives in this study. 
a
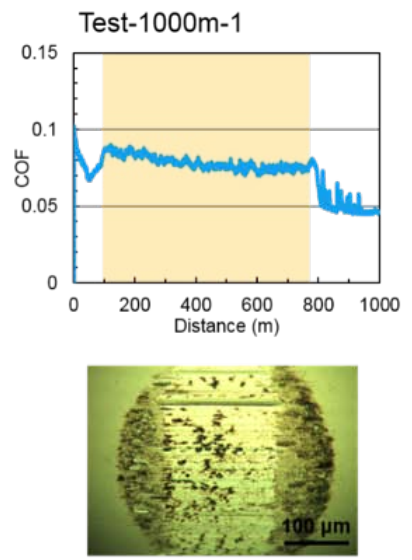

b

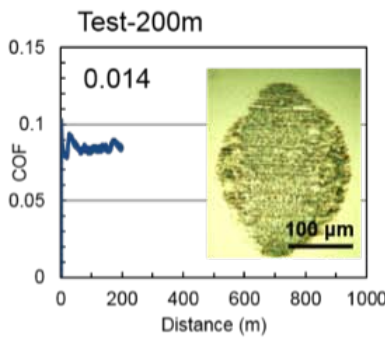

Test-1000m-2
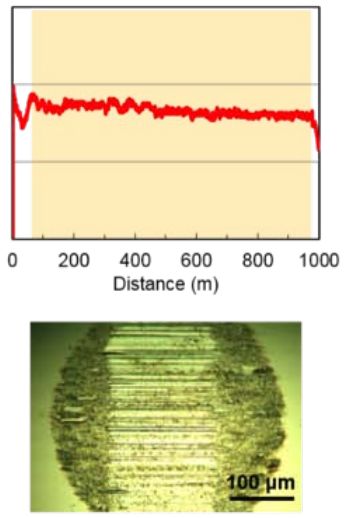

Test-1000m-3
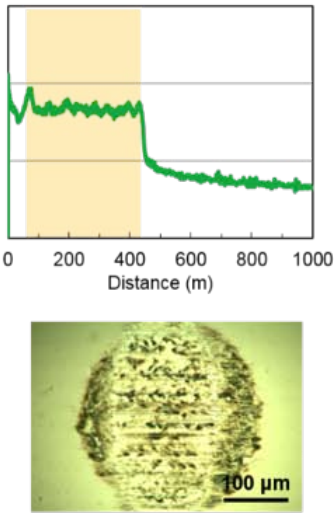

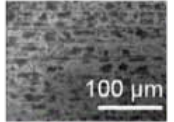

O
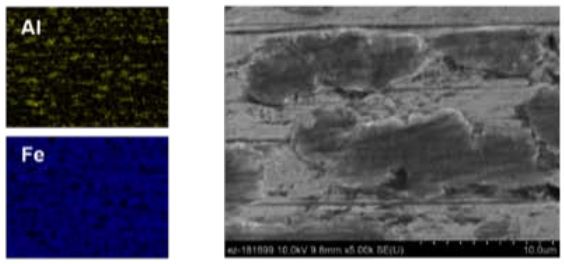

C

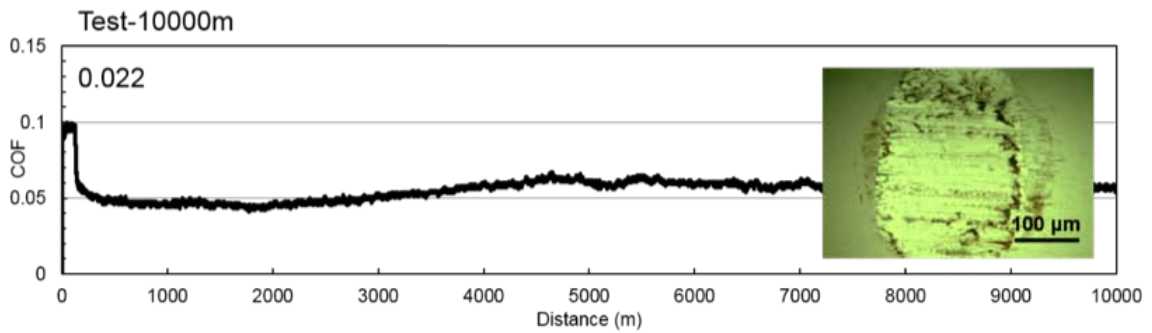

Figure 2. Friction and wear results of the Al 380 alloy flats sliding against 52100 steel balls in the base oil. (a) Coefficient of friction (COF) of 1,000 m sliding tests and the optical images of steel ball wear scars. (b) COF of a 200 m sliding test and the SEM images and EDS maps of the steel ball wear scar suggesting that the transfer film is rich in aluminum and oxygen. (c) COF of a $10,000 \mathrm{~m}$ sliding test and an optical image of the steel ball wear scar with little transfer material.

The wear protection on the $\mathrm{Al} 380$ alloy is ranked as ZDDP > carboxylate IL > phosphate IL. In contrast, the sulfonate IL surprisingly caused an increase in friction (steady-state COF around 0.12) and significant material loss $\left(\sim 5.0 \mathrm{~mm}^{3}\right)$, much higher than that for the base oil without the sulfonate IL. Figure $3 \mathrm{c}$ plots the wear volume of individual tests against the sliding distance of highfriction stage $(\mathrm{COF}>0.065)$, which shows a strong linear correlation.

The sulfonate IL was further investigated because of its wear problem in lubricating Al described above. There was no sign of corrosion on the Al 380 alloy surface after being exposed to the sulfonate IL for two weeks at room temperature or $2 \mathrm{~h}$ at $200^{\circ} \mathrm{C}$. The sulfonate IL was then added to the base oil at various concentrations, contributing 200 ppm P, 80 ppm P, and 33 ppm P, respectively. All concentrations behaved similarly and the reduction in concentration did not seem to slow down the material removal rate, as shown in Figure 4. One hypothesis for the detrimental effect is tribocorrosion. Sulfonate IL is known to quickly react with the aluminum surface to form a passive film in static environments [4]. In tribo-contact, however, such a passive layer would be damaged or removed instantaneously by the wear process, which will expose the fresh substrate to trigger new reactions such a mechanochemical process is suspected to cause an increased material removal rate. 
a
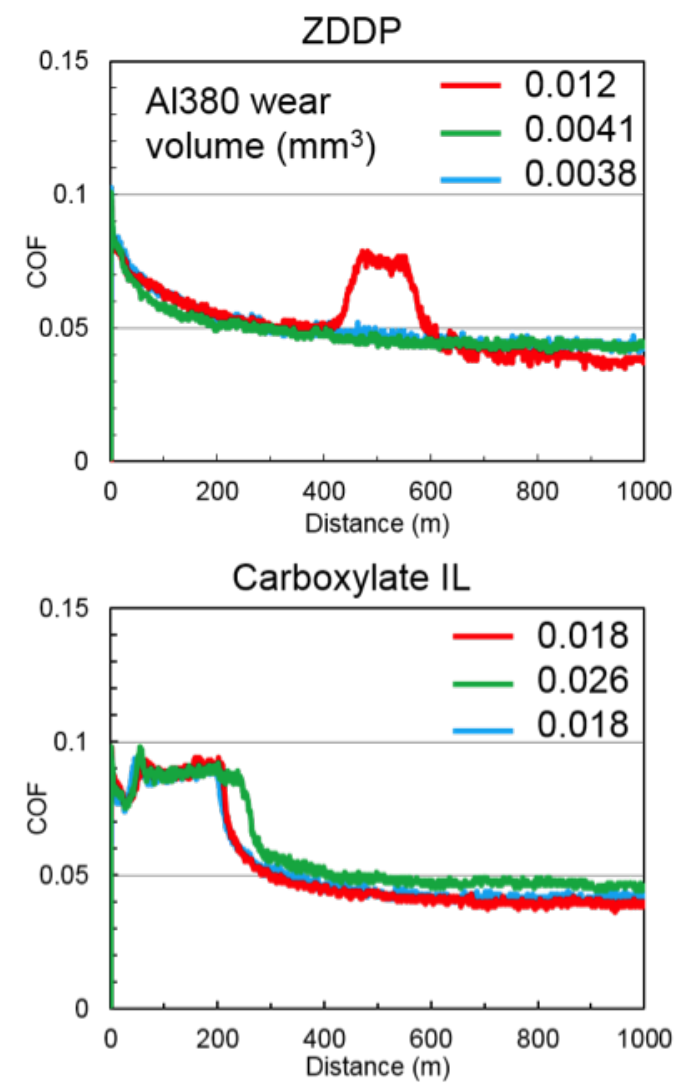

Phosphate IL

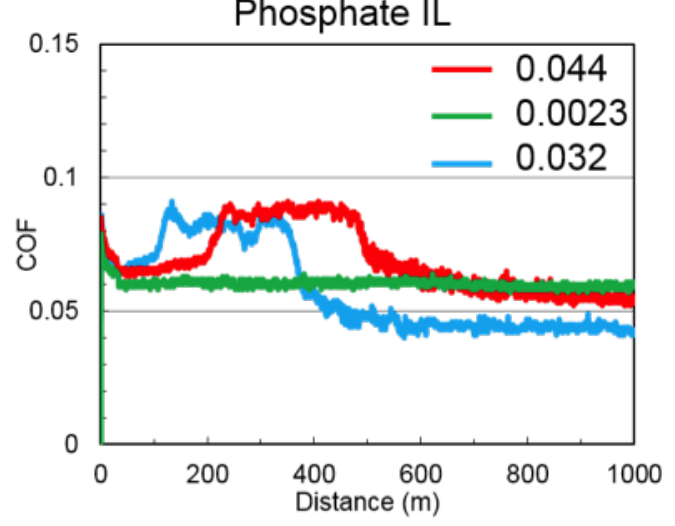

Sulfonate IL

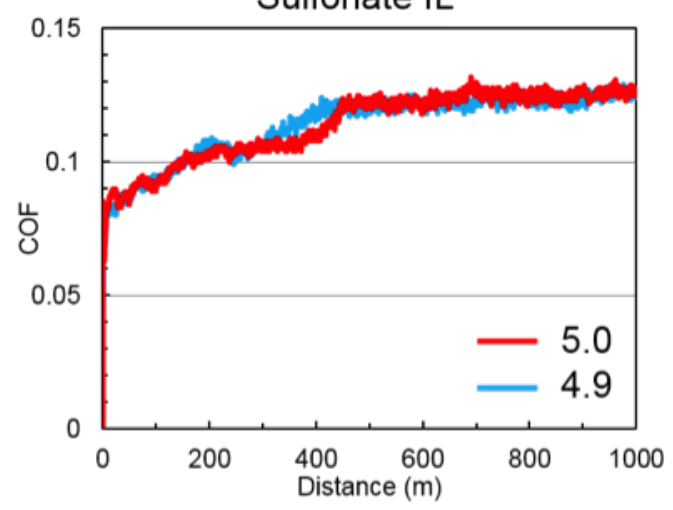

b

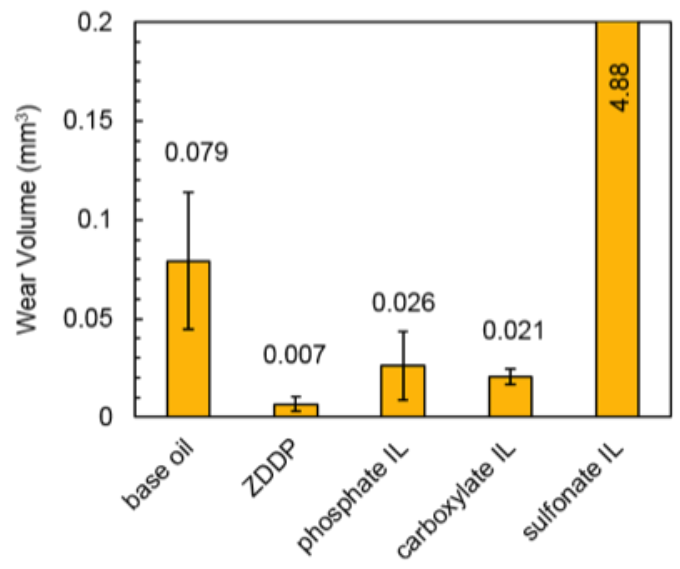

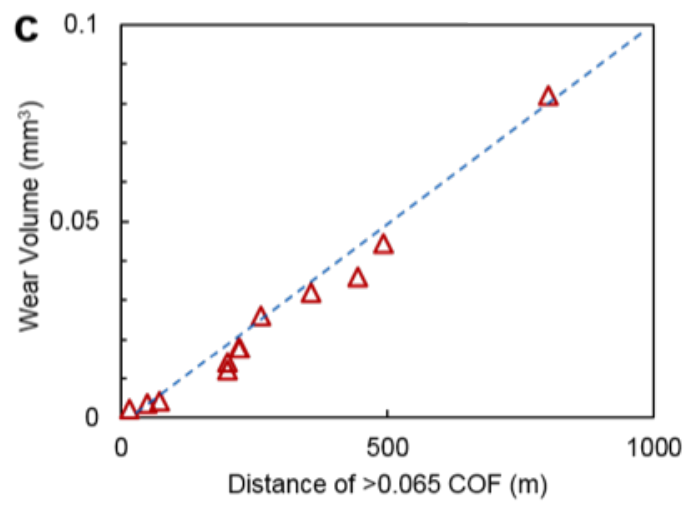

Figure 3. Friction and wear results of the Al 380 alloy flats sliding against 52100 steel ball in the oils containing a ZDDP or IL. (a) Friction behavior of ZDDP, phosphate IL, carboxylate IL, and sulfonate IL at 800 ppm P. Wear volumes were marked in the legend in $\mathrm{mm}^{3}$. (b) Comparison of wear volumes. (c) A plot of the wear volume of individual test vs. the sliding distance for tests having COF $>0.065$.

SEM images and EDS spectra of the worn Al 380 surfaces are shown in Figure 5. There were no significant peaks for $\mathrm{Zn}$, P, or S, suggesting the lack of detectable tribofilm formed by either ZDDP or ILs. Further investigation will be carried out by using the surface-sensitive X-ray photoelectron spectroscopy.
Figure 6 shows the friction traces and wear volumes for the combinations of ZDDP+IL (1:1 molecular ratio) at a total $800 \mathrm{ppm}$ P. Interestingly, the detrimental effect of the sulphonate IL was mitigated by the ZDDP when used together. On the other hand, an antagonistic effect was discovered between the ZDDP and carboxylate IL with 

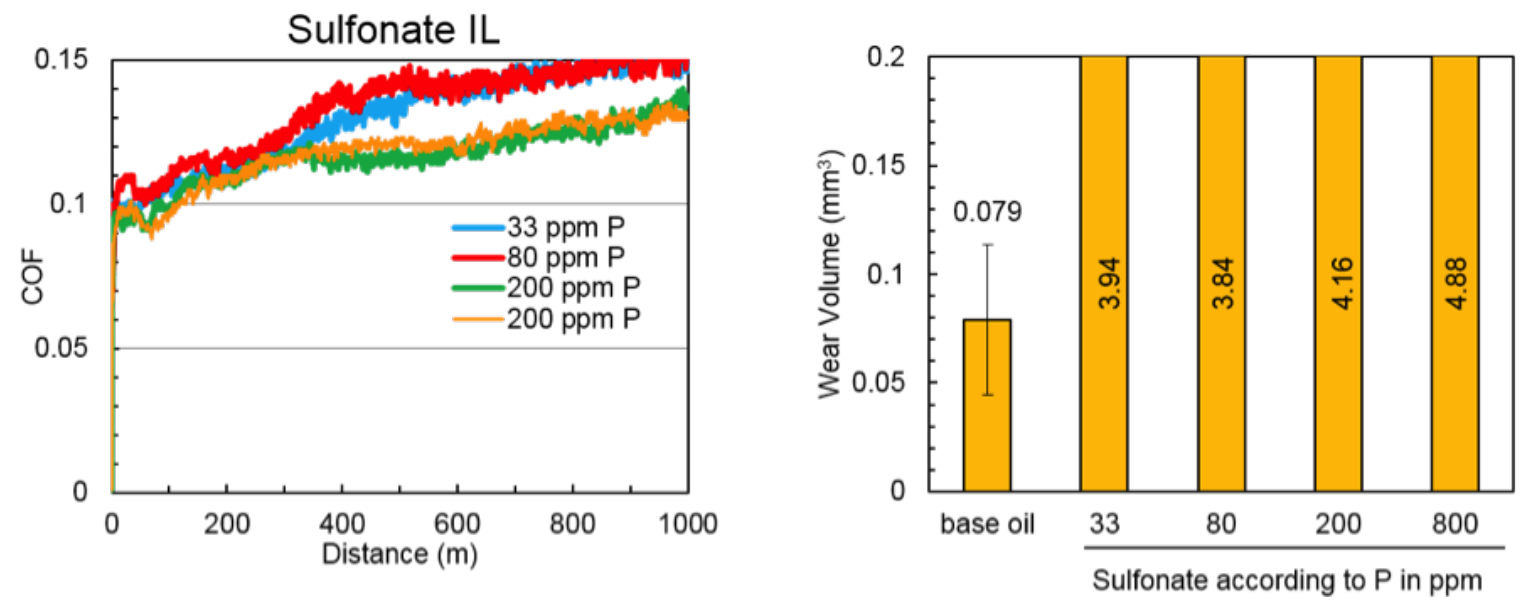

Figure 4. Friction traces and wear volumes of Al 380 alloy flat sliding against 52100 steel ball in the oils containing various amounts of the sulfonate IL with P contents of 200 ppm, 80 ppm, and 33 ppm.

Base oil
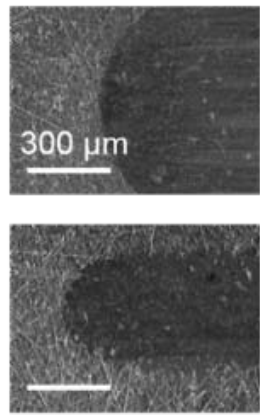

ZDDP

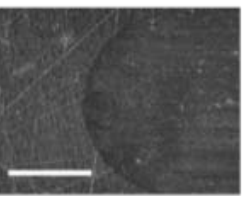

Phosphate IL

Carboxylate IL
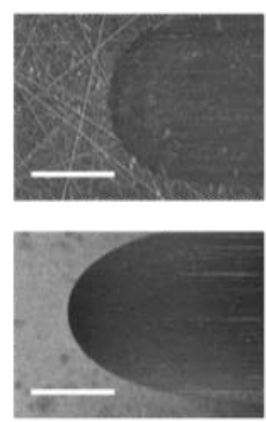
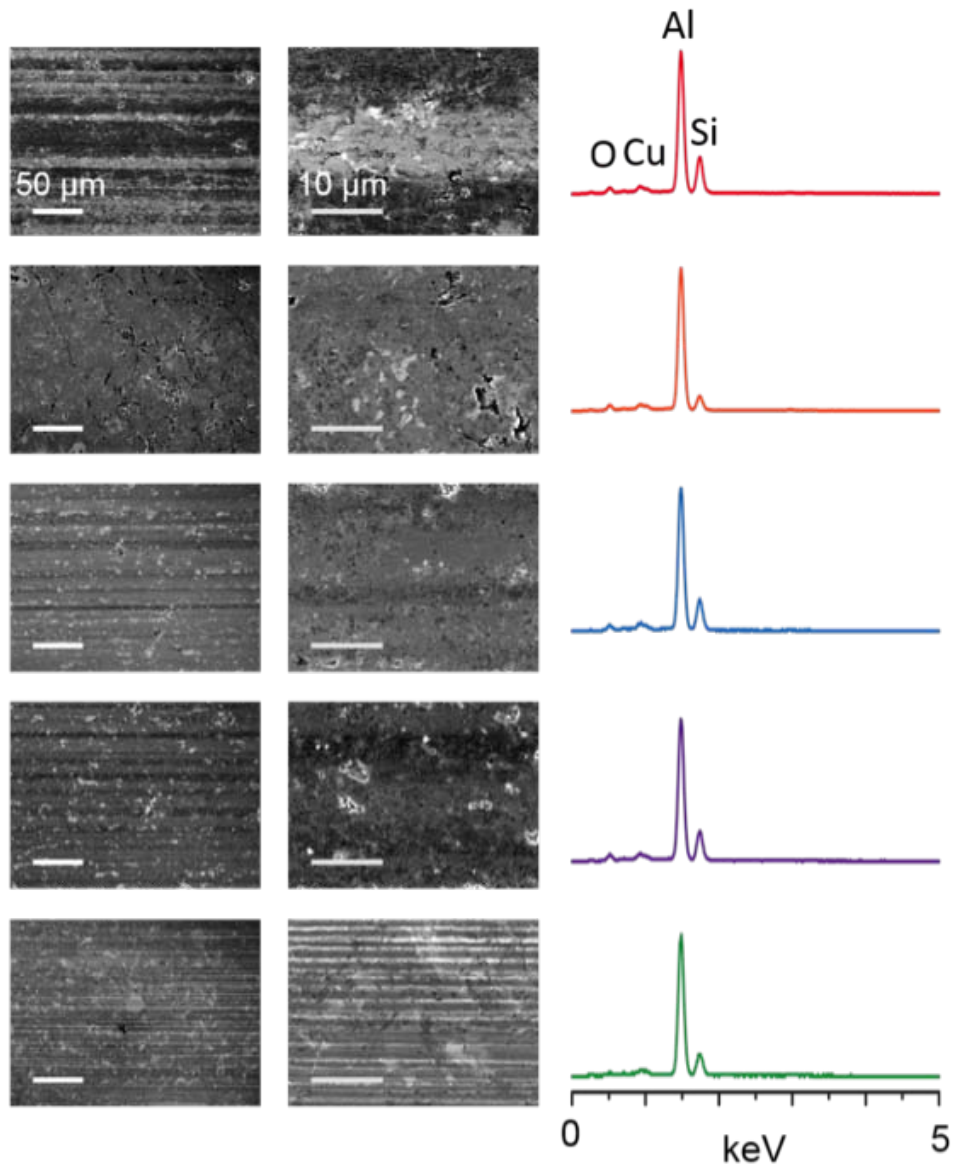

Figure 5. SEM images and EDS spectra of the wear scars on AI 380 alloy flat.

wear rate increased by more than two orders of magnitude compared with using either of them alone. Again, tribocorrosion is a possible explanation. While carboxylic acid alone has little corrosive attack to aluminum, the combination of carboxylic acid and phosphate has been used for efficient dealumination [5]. This suggests potentially strong chemical reactions with aluminum when the ZDDP and carboxylate IL both are present. 

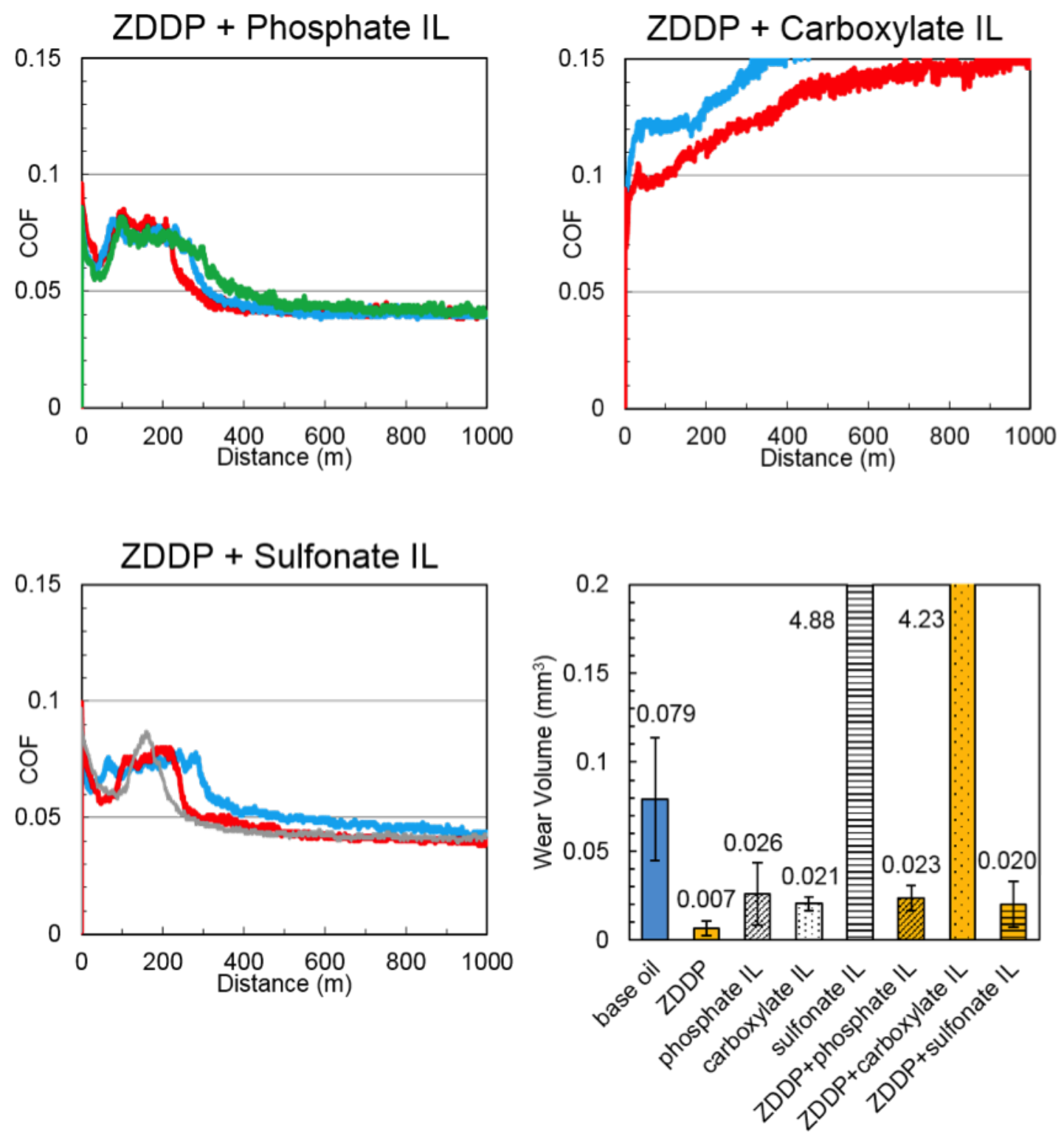

Figure 6. Friction and wear results of the Al 380 alloy flats sliding against 52100 steel ball in the oils containing the ZDDP and an IL at 1:1 molecular ratio with the total P content of $800 \mathrm{ppm}$.

\section{Investigation of the Compatibility Between AW and Bronze}

We have started the study of compatibility between AW additives and bronze. However, initial wear results showed poor repeatability from sample to sample. For the same lubricant, the wear on one bronze sample could be several times higher than that of another bronze sample, whereas all bronze samples were machined from the same plate. Investigation is under way.

\section{Conclusions}

A series of boundary lubrication tribo-tests have been conducted on selected Al-Si and bronze alloys against steel using ZDDP- and IL-additized lubricants. The friction and wear behavior varied significantly for different additive chemistry. In lubricating the Al 380 alloy, the addition of ZDDP, phosphate IL, carboxylate IL, ZDDP+phosphate IL or ZDDP+sulfonate IL provided effective wear protection. However, the sulfonate IL and mixture of ZDDP and carboxylate IL had unexpected detrimental impact increasing the wear rate by two orders of magnitude. A tribo-corrosion hypothesis has been 
proposed and characterization is being performed to seek fundamental understanding. Investigation is underway for the poor repeatability observed in the tests of the bronze alloy.

\section{References}

1. Kawamura, M.; Fujita, K., "Antiwear property of lubricant additives for high silicon aluminum alloy under boundary lubricating conditions," Wear 89 (1983), 99-105.

2. Wan, Y.; Cao, L. L.; Xue, Q. J., "Friction and wear characteristics of ZDDP in the sliding of steel against aluminum alloy," Tribology International 30 (1997), 767-772.

3. Y. Zhou, J. Dyck, T. Graham, H. Luo, D.N. Leonard, J. Qu, "Ionic liquids composed of phosphonium cations and organophosphate, carboxylate, and sulfonate as lubricant antiwear additives," Langmuir 30 (2014) 13301-13311.

4. B. Zhou, Y. Wang, Y. Zuo, "Evolution of the corrosion process of AA 2024-T3 in an alkaline $\mathrm{NaCl}$ solution with sodium dodecylbenzenesulfonate and lanthanum chloride inhibitors," Applied Surface Science 357 (2015) 735-744.

5. X. Chang, L. He, H. Liang, X. Liu, Z. Yan, "Screening of optimum condition for combined modification of ultra-stable Y zeolites using multi-hydroxyl carboxylic acid and phosphate," Catalysis Today 158 (2010) 198-204.

\section{FY 2016 Publications/Presentations}

1. J. Qu, "Using ionic liquids as anti-wear additives to lubricate non-metallic surfaces," $20^{\text {th }}$ International Colloquium Tribology, Stuttgart, Germany, January 12-14, 2016. (Invited)

2. J. Qu, T. Toops, "Ionic liquids as engine lubricant additives, impact on emission control catalysts, and compatibility with coatings," DOE Vehicle Technologies Program Annual Merit Review, Washington, D.C., June 6-9, 2016.

3. Y. Zhou, J. Qu, "Investigation of the compatibility between anti-wear additives and non-ferrous bearing alloys," STLE Tribology Frontiers Conference, Chicago, November 13-15, 2016. 